\title{
Oxaliplatin-induced Pulmonary Fibrosis: Two Case Reports
}

\author{
Chun-Geun Ryu, Eun-Joo Jung, Gangmi Kim, Su Ran Kim, Dae-Yong Hwang \\ Department of Surgery, Colorectal Cancer Center, Konkuk University Medical Center, Konkuk University School of Medicine, Seoul, Korea
}

Oxaliplatin with infusional 5-fluorouracil plus leucovorin (FOLFOX regimen) is the one of the standard chemotherapy regimens for treating a colorectal carcinoma. The most common side effects include neutropenia, diarrhea, vomiting and peripheral neuropathy, and these are moderate and manageable. However, pulmonary toxicity is rarely reported to be associated with the FOLFOX regimen. Moreover, there is no established guideline for the management of this side effect. Here, along with a literature review, we report two cases of rapidly developing pulmonary fibrosis related to the use of the FOLFOX regimen in patients with colorectal carcinomas.

Keywords: Oxaliplatin; Toxicity; Pulmonary fibrosis

\section{INTRODUCTION}

Oxaliplatin-based chemotherapy, including the FOLFOX regimens has been widely used to treat colorectal cancer. However, there have been rare reports of pulmonary toxicity associated with FOLFOX regimen. Here, we report two cases of rapidly developing to pulmonary fibrosis related to FOLFOX regimen usage in colorectal carcinoma.

\section{CASE REPORTS}

\section{Case 1}

A 55-year-old man had visited another hospital in May 2010 with presenting symptoms of low abdominal pain and bowel habit change for several months. He had no history of smoking, hypertension, diabetes mellitus, hepatitis or pulmonary disease. Colonoscopy showed a recto-sigmoid colon tumor. Colonoscopic biopsy revealed a moderately differentiated adenocarcinoma. On computed tomography (CT) and positive emission tomography (PET)/

Received: September 7, 2011 Accepted: October 10, 2011

Correspondence to: Dae-Yong Hwang, M.D.

Department of Surgery, Colorectal Cancer Center, Konkuk University Medical Center, Konkuk University School of Medicine, 4-12 Hwayang-dong,

Gwangjin-gu, Seoul 143-729, Korea

Tel: +82-2-2030-5111, Fax: +82-2-2030-5112

E-mail: hwangcrc@kuh.ac.kr

(c) 2011 The Korean Society of Coloproctology

This is an open-access article distributed under the terms of the Creative Commons Attribution NonCommercial License (http://creativecommons.org/licenses/by-nc/3.0) which permits unrestricted noncommercial use, distribution, and reproduction in any medium, provided the original work is properly cited.
CT, the tumor was shown to be located on the recto-sigmoid junction, with multiple lymph-node metastases on the left supra-clavicular, para-vertebral and para-aortic areas and with no hepatic or pulmonary metastasis. Under the diagnosis of recto-sigmoid colon cancer with multiple distant metastases of lymph nodes, a laparoscopic-assisted anterior resection was performed in that hospital. The pathology result for a specimen showed a moderately differentiated adenocarcinoma that had infiltrated the pericolic adipose tissue. Lymphatic, perineural and venous invasions were presented. Metastases of regional lymph nodes were found in 16 out of the 20 retrieved pericolic lymph nodes. The final pathologic stage was T3N2bM1, stage IV. After recovering from surgery, the patient visited our hospital for palliative treatment. FOLFOX-4 regimen (oxaliplatin with infusional 5-fluorouracil plus leucovorin)[1] was chosen and started in June 2010; targeted agents were not included because of the economic situation of the patient. Except for intermittent grade- 3 neutropenia, the patient tolerated FOLFOX-4 chemotherapy well.

On the follow-up CT after the 12th cycle of chemotherapy, the multiple lymph-node metastases on the left supra-clavicular, paravertebral, and para-aortic areas had disappeared. Two weeks following the end of 13th cycle of chemotherapy, dry cough and dyspnea developed, and the symptoms rapidly worsened. On the 28th day after 13th cycle of chemotherapy, he visited the hospital due to pulmonary symptoms. Vital signs were stable with normal body temperature. On physical examination, dyspnea on exertion was presented, and inspiratory crackles were audible on both lungs. There was no chest retraction, wheezing or cyanosis. On arterial blood gas analysis (ABGA), a pH of 7.41, a $\mathrm{PaCO}_{2}$ of $27.8 \mathrm{mmHg}$ and a $\mathrm{PaO}_{2}$ of $86.9 \mathrm{mmHg}$ were shown in room air. The chest X- 
ray and high-resolution chest computed tomography (HRCT) demonstrated parenchymal distorsion, fibrosis and multiple patchy ground-glass opacities on both lungs (Fig. 1).

Under the suspicion of oxaliplatin-induced pulmonary toxicity, idiopathic pulmonary fibrosis, he was admitted and received broadspectrum antibiotics and oral corticosteroid immediately. For the differential diagnosis with infectious pneumonia and malignant

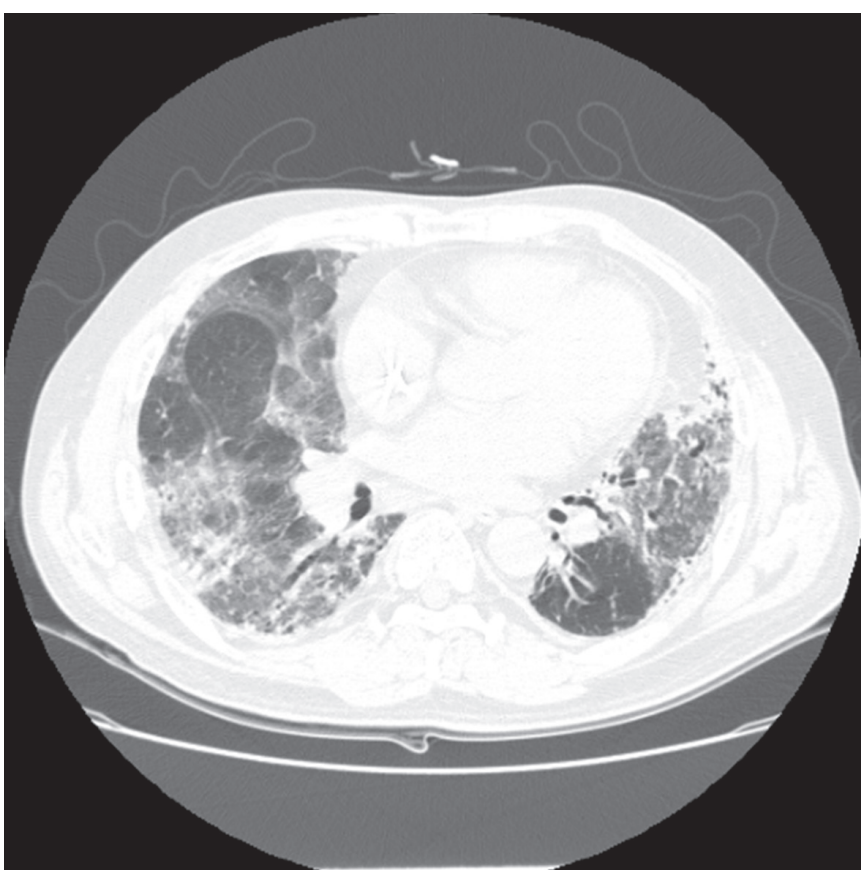

Fig. 1. High-resolution chest computed tomography showing the parenchymal distorsion, the fibrosis and multiple patchy ground-glass opacities on both lungs.

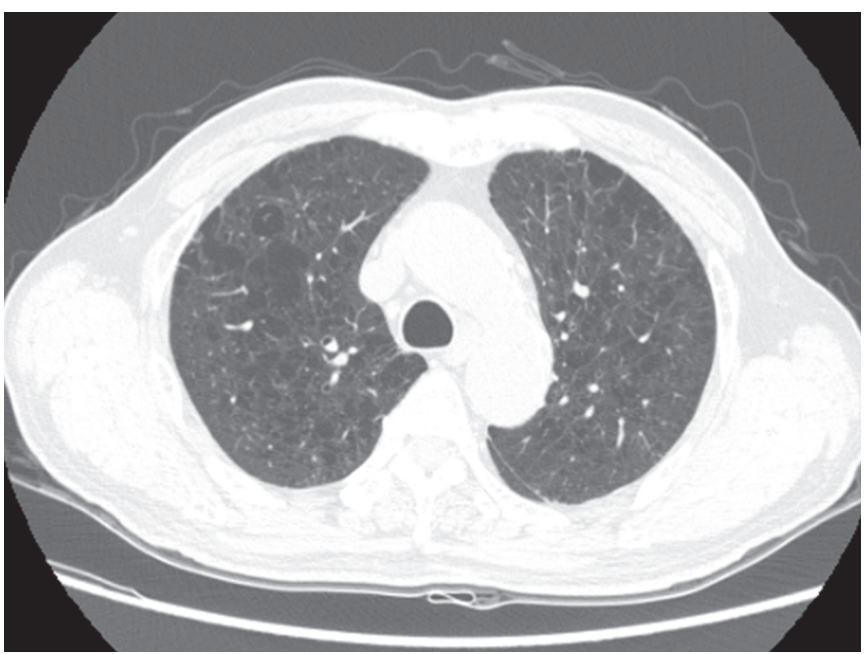

Fig. 2. High-resolution chest computed tomography before the initiation of oxaliplatin, 5-fluorouracil, and leucovorin (FOLFOX) treatment. There is underlying emphysema on both upper lungs. disease, bronchoscopy was performed. On bronchoscopic examination, there was no endobronchial lesion, and no other infection or malignancy was shown on bronchoscopic biopsy or bronchoalveolar lavage cytology. The bronchial washing culture was also negative. At the beginning of the treatment, the patient had felt better in terms of dyspnea. However, after two days of hospitalization, the dyspnea worsened; in spite of the medical treatment, the diffuse ground-glass opacities the on chest X-ray had progressed. On the 3rd hospital day, the patient was moved into the intensive care unit (ICU) and intubated, and mechanical ventilation was started. Steroid therapy was changed to intravenous methylprednisone, but the patient showed a poor clinical course and progressively worsening radiographic findings. On the $23 \mathrm{rd}$ hospital day, he died due to irreversible respiratory failure and multiple organ failure.

\section{Case 2}

A 73-year-old man with hepatic flexure colon cancer was referred to the Department of Surgery for operation. He had a medical history of noninsulin-dependent diabetes mellitus without complications and no history of cigarette smoking. A right hemicolectomy was performed for colon cancer on the hepatic flexure in July 2009. Pathology showed a moderately differentiated adenocarcinoma that had infiltrated the pericolic adipose tissue. Lymphatic, perineural and venous invasions were not presented. Metastasis of a regional lymph node was found in 1 out of 34 retrieved pericolic lymph nodes. The final pathologic stage was T3N1M0, stage IIIB. A chest X-ray and chest CT before the initiation of chemotherapy showed an emphysematous lung on both upper lobes and the right middle lobe, but no pulmonary symptoms were present (Fig. 2). Adjuvant chemotherapy, including the FOLFOX-4 regimen, was started in September 2009. There were no specific changes on follow up after every three cycles of chemotherapy, including CT and

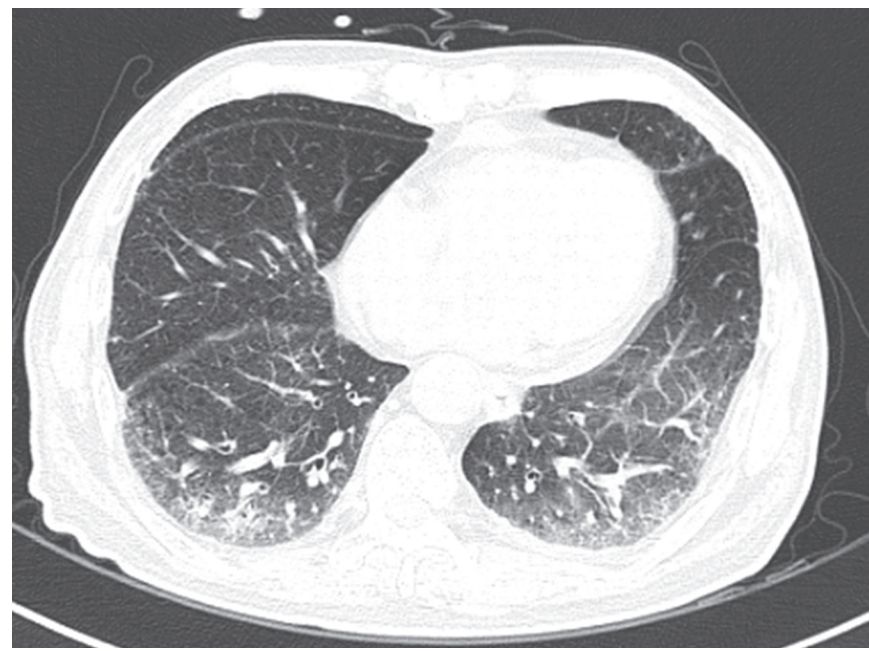

Fig. 3. High-resolution chest computed tomography. There are groundglass areas on both lower lungs and a patchy consolidation on the right lower lung. 
tumor markers, during the course of the treatment.

During the 9th cycle of the FOLFOX-4 regimen, immediately after the administration of oxaliplatin, dyspnea suddenly developed on the 1st hospital day. At the time, the body temperature was $39.3^{\circ} \mathrm{C}$. Pulmonary auscultation revealed inspiratory crackles in both lungs, and dyspnea on exertion was presented. Leukocytosis $\left(23.63 \times 10^{3} / \mu \mathrm{L}\right)$ with a predominance of neutrophils $(94.5 \%)$ was noted on the laboratory test. C-reactive protein was elevated at $16.89 \mathrm{mg} / \mathrm{dL}$ (reference range, $<0.5 \mathrm{mg} / \mathrm{dL}$ ). A chest X-ray and HRCT showed ground-glass opacities in the left upper lobe and both lower lobes, and a patchy consolidation in the right middle lobe (Fig. 3). A treatment with oxygen therapy with a mask, corticosteroids and broad-spectrum antibiotics were started. Sputum and blood cultures were negative. On the test for the Swine influenza A virus, which we conducted because of a flu epidemic at the time, virus infection was negative. Based on the clinical course and imaging findings, we made a diagnosis of FOLFOX-induced interstitial pneumonitis or hypersensitivity pneumonia. He presented with progressive respiratory failure and was transferred to the ICU for mechanical ventilation on the 11th hospital day. The clinical course was unfavorable, with worsening of respiratory insufficiency, leading to the patient's death on the 18th hospital day.

\section{DISCUSSION}

Oxaliplatin-containing regimens, including the FOLFOX regimens have become a part of the standard treatment in a high number of colorectal-cancer patients with regional lymph-node metastasis for adjuvant setting and advanced stage [2]. The major toxicity of oxaliplatin is a peripheral neuropathy. Renal, haematological and gastrointestinal toxicities are less frequent [3]. To our knowledge, oxaliplatin-induced pulmonary toxicity is rare [4], so there is no established guideline for its management.

We experienced two fatal cases with interstitial pneumonitis rapidly progressing to pulmonary fibrosis during FOLFOX-4 chemotherapy. Muneoka et al. [5] reported a case of FOLFOX-induced interstitial pneumonitis, which did not recur after the reintroduction of 5-fluorouracil/leucovorin alone. However, Yague et al. [3] reported interstitial lung disease after oxaliplatin monotherapy (85$\mathrm{mg} / \mathrm{m}^{2} \mathrm{D} 1$ intravenous in a 2 -hour infusion every 3 weeks). Therefore, the main cause of FOLFOX-regimen-induced interstitial pneumonitis is thought to be oxaliplatin. Several case reports of oxaliplatin-induced pulmonary fibrosis have been reported. The details of those cases are shown in Table 1. The manifestations of druginduced pulmonary toxicity are usually nonspecific, so an accurate diagnosis is difficult.

Once oxaliplatin-induced interstitial pneumonitis has occurred, cessation of oxaliplatin is mandatory [5]. High-dose corticosteroid treatment is commonly given for serious cases. However, the role of high-dose corticosteroid treatment in the management of oxaliplatin-induced interstitial pneumonitis remains unclear [5]. Of the 14 cases reported in Table 1, 13 patients were treated with highdose corticosteroid, but 5 of the treated patients died. The median number of cycles of FOLFOX and the median dose of oxaliplatin were 6 cycles (range, 4 to 6 cycles) and $510 \mathrm{mg} / \mathrm{m}^{2}$ (range, 340 to $\left.780 \mathrm{mg} / \mathrm{m}^{2}\right)$ in the patients who died and 13 cycles and $1,105 \mathrm{mg} /$

Table 1. Reported cases of oxaliplatin-induced pulmonary fibrosis

\begin{tabular}{|c|c|c|c|c|c|c|c|}
\hline & Author & Sex/Age & No. of cycles & $\begin{array}{c}\text { Total dose } \\
\left(\mathrm{mg} / \mathrm{m}^{2}\right)\end{array}$ & Primary lesion & Steroid treatment & Result \\
\hline 1 & Trisolini et al. [8] & $\mathrm{M} / 60$ & 7 & 910 & Rectum & Given & Improvec \\
\hline 2 & Gagnadoux et al. [6] & $\mathrm{F} / 60$ & 8 & 680 & Colon & None & Improved \\
\hline 3 & Yague et al. [3] & $\mathrm{F} / 68$ & 6 & 780 & Colon & Given & Dead \\
\hline 4 & Ruiz-Casado et al. [9] & $\mathrm{M} / 67$ & 11 & 1,100 & $?$ & Given & Improved \\
\hline 5 & Pasetto and Monfardini [4] & $\mathrm{M} / 74$ & 6 & 510 & Sigmoid & Given & Dead \\
\hline 6 & Garrido et al. [10] & $\mathrm{F} / 30$ & 6 & 510 & Rectum & Given & Improved \\
\hline 7 & Arevalo et al. [2] & $\mathrm{M} / 73$ & 4 & 340 & Sigmoid & Given & Dead \\
\hline 8 & Arevalo et al. [2] & $\mathrm{M} / 71$ & 4 & 340 & Sigmoid & Given & Dead \\
\hline 9 & Wilcox et al. [7] & $\mathrm{M} / 71$ & 6 & 510 & $?$ & Given & Dead \\
\hline 10 & Wilcox et al. [7] & $\mathrm{M} / 77$ & 12 & 1,020 & Colon & Given & Improved \\
\hline 11 & Wilcox et al. [7] & $\mathrm{M} / 69$ & 6 & 510 & Colon & Given & Improved \\
\hline 12 & Ohori et al. [11] & M/69 & 10 & 850 & Colon & Given & Improved \\
\hline 13 & Ohori et al. [11] & $\mathrm{M} / 72$ & 11 & 935 & Colon & Given & Improved \\
\hline 14 & Muneoka et al. [5] & M/82 & 10 & 850 & Colon & Given & Improvec \\
\hline 15 & Case 1 & M/55 & 13 & 1,105 & RS & Given & Dead \\
\hline 16 & Case 2 & $\mathrm{M} / 73$ & 8 & 680 & $\mathrm{HF}$ & Given & Dead \\
\hline
\end{tabular}

$\mathrm{RS}$, rectosigmoid; HF, hepatic flexure. 
$\mathrm{m}^{2}$ in case 1 and 8 cycles and $680 \mathrm{mg} / \mathrm{m}^{2}$ in case 2 , respectively. Although there were slight differences in the median numbers of cycles of FOLFOX and the median doses of oxaliplatin, some cases had a rapid and untoward course $[2-4,6,7]$, similar to what occurred in our cases.

Our patients had a rapidly progressive respiratory insufficiency, needed mechanical ventilation on the 3rd day of admission in case 1 and on the 8th day of admission in case 2, were refractory to conventional high-dose corticosteroid treatment, had a poor clinical and radiographic course, and eventually died on the 23rd hospital day in case 1 and on the 18th hospital day in case 2 respectively. Even though interstitial pneumonitis is rare in patients treated with the FOLFOX regimen, we recommend careful monitoring of drug-induced pulmonary toxicity, which may occur in patients during or at the end of oxaliplatin chemotherapy.

\section{CONFLICT OF INTEREST}

No potential conflict of interest relevant to this article was reported.

\section{REFERENCES}

1. Andre T, Boni C, Mounedji-Boudiaf L, Navarro M, Tabernero J, Hickish T, et al. Oxaliplatin, fluorouracil, and leucovorin as adjuvant treatment for colon cancer. N Engl J Med 2004;350:2343-51.

2. Arevalo Lobera S, Sagastibeltza Marinelarena N, Elejoste Echeberria I, Mele Olive M, Egana Otano L, Basterretxea Badiola L, et al. Fatal pneumonitis induced by oxaliplatin. Clin Transl Oncol 2008;10:764-7.
3. Yague XH, Soy E, Merino BQ, Puig J, Fabregat MB, Colomer R. Interstitial pneumonitis after oxaliplatin treatment in colorectal cancer. Clin Transl Oncol 2005;7:515-7.

4. Pasetto LM, Monfardini S. Is acute dyspnea related to oxaliplatin administration? World J Gastroenterol 2006;12:5907-8.

5. Muneoka K, Shirai Y, Sasaki M, Wakai T, Sakata J, Hatakeyama K. Interstitial pneumonia arising in a patient treated with oxaliplatin, 5-fluorouracil, and, leucovorin (FOLFOX). Int J Clin Oncol 2009;14:457-9.

6. Gagnadoux F, Roiron C, Carrie E, Monnier-Cholley L, Lebeau B. Eosinophilic lung disease under chemotherapy with oxaliplatin for colorectal cancer. Am J Clin Oncol 2002;25:388-90.

7. Wilcox BE, Ryu JH, Kalra S. Exacerbation of pre-existing interstitial lung disease after oxaliplatin therapy: a report of three cases. Respir Med 2008;102:273-9.

8. Trisolini R, Lazzari Agli L, Tassinari D, Rondelli D, Cancellieri A, Patelli M, et al. Acute lung injury associated with 5-fluorouracil and oxaliplatinum combined chemotherapy. Eur Respir J 2001; 18:243-5.

9. Ruiz-Casado A, Garcia MD, Racionero MA. Pulmonary toxicity of 5-fluoracil and oxaliplatin. Clin Transl Oncol 2006;8:624.

10. Garrido M, O’Brien A, Gonzalez S, Clavero JM, Orellana E. Cryptogenic organizing pneumonitis during oxaliplatin chemotherapy for colorectal cancer: case report. Chest 2007;132:1997-9.

11. Ohori H, Takahashi M, Ogasawara N, Suzuki M, Miyate Y, Kato S. Two cases of interstitial lung diseases in patients treated with oxaliplatin, 5-fluorouracil and Leucovorin (FOLFOX). Gan To Kagaku Ryoho 2009;36:295-8. 